\title{
Microgrid Connection Management based on an Intelligent Connection Agent
}

\author{
J. Rocabert ${ }^{1}$, Student Member, IEEE, G. Azevedo ${ }^{2}$, Student Member, IEEE, I. Candela ${ }^{1}$, Member, IEEE, \\ R. Teoderescu ${ }^{3}$, Member, IEEE, P. Rodriguez ${ }^{1}$, Member, IEEE, I. Etxebarria-Otadui ${ }^{4}$, Member, IEEE. \\ ${ }^{1}$ Electrical Engineering \\ Department \\ Technical University of \\ Catalonia \\ (UPC) Terrassa, Spain \\ ${ }^{2}$ Department of Electrical \\ Engineering \\ Federal University of \\ Pernambuco (UFPE) \\ Recife, Pernambuco Brazil \\ ${ }^{3}$ Institute of Energy \\ Technology IET, Aalborg \\ University AAU, \\ Denmark. \\ ${ }^{4}$ IKERLAN-IK4 \\ Technological \\ Research Center, \\ Spain
}

Contac Author:

rocabert@ee.upc.edu

\begin{abstract}
The increasing penetration of generation power plants based on renewable energies in the electrical networks has boosted the number of systems connected to the grid. In this scenario the microgrid, providing advanced functionalities to improve the stability and operation of the network, have become very popular. In this paper a control strategy for the microgrid management is presented. The proposed system improves the performance of microgrids and its interaction with the main network, or another microgrid, under grid voltage transients. This technique gives rise to a simple and robust control scheme to ensure the appropriate microgrid disconnection, and further resynchronization and reconnection, from the main grid. In the following, the control algorithm for the intelligent connection agent will be shown, and the grid synchronization system, as well as the voltage and current control loops will be detailed. Finally, results obtained using the MATLAB/Simulink \& PSIM platform will be presented and discussed.
\end{abstract}

\section{INTRODUCTION}

The integration of Distributed Generation is increasing significantly in the last years, mainly, due to the integration of photovoltaic and wind power plant in medium and low voltage grids. Lately, storage energy systems are being integrated into the electrical grid with the aim of improving stability and quality service.

Is usual the use of renewable sources and other small scale in distributed generators systems equipped with Voltage Source Inverter (VSI) or Current Sources Inverters (CSI). Similarly, in Uninterruptible Power Supplies (UPS) applications, parallel redundant VSI are used to increase the reliability of the electrical network. In a near future, a lot of distributed generators with dc-ac converters will feed the utility networks, so a good performance of those under any electric grid situation must be guaranteed. Until now antiislanding techniques has been used to disconnect distributed generators in front of grid faults, as in [1] - [3]. But a new set of specifications will be required for the proper performance of the inverters in network failure situations. One of them will be the capability to work in island grids, where could be available to ensure energy continuity with the critical loads.
There are several references in the literature dealing with connection and disconnection of microgrids from the main electrical network. For instance, the implementation of an intelligent and autonomous switch, is presented in [4]. This system is based on monitoring the operating conditions of both the microgrid and the main network and, depending on the status of the main network, the microgrid disconnection or reconnection procedure is triggered.

Synchronization of isolated microgrid is usually accomplished by detecting the difference in the phase between the mains and the microgrid. In [5] two synchronization PLL loops are required to achieve the resynchronization. One designated to detect the microgrid phase voltage and the other one to main grid phase. When the mains is restored and the microgrid still works in island mode the mains phase angle is detected. The difference between both phase angles is minimized conducting to zero the microgrid voltage quadrature-component through a PIcontroller. Taking only the quadrature voltage from the mains, one PLL can be removed and work with only the phase seen from the microgrid voltage.

In this paper, a VSI working as a grid former is purposed. It will work as an observer when the grid is on, but under any failure disconnects the microgrid from the main grid and gives a nominal voltage reference for the difference CSI connected in it. Under a power unbalance, the VSI has the capability to give the transient power while a higher level low-bandwidth control determines the new reference of each CSI to restore the new steady-state point. One of the most difficult transient is in the reconnection process with the main grid, so a slow resynchronization process must be done. The transition between these two operation modes should be as transparent and fast as be possible, in order to maximize the quality in supplying loads connected to the microgrid.

In this paper a solution for transients and steady-stage control from grid connected to islanded microgrids will be proposed, and also its posteriori reconnection when the main 
grid is available. In the scope of the paper, a primary control method is presented to achieve a fast transient and a good operability both in grid connected mode and island mode. This strategy presented in the paper is evaluated through simulations.

\section{MICROGRID OPERATION MODE}

In this section the two main operation modes of a microgrid, grid connected and islanded mode, are explained. The Intelligent Connection Agent (ICA) is responsible of switching to the most appropriate operation mode. However, this decision depends mainly on the network conditions.

Fig. 1 shows the utility and the microgrid considered in the purpose of this paper. In this scenario, the microgrid is composed by the main switch, ICA, the grid-forming VSI, different CSI working as grid feeding converters and different loads. As shown in Fig.1, the VSI, takes charge of processing the energy delivered/stored by the microgrid, and a controlled switch $\left(S_{i}\right)$, which physically connects the microgrid to the main grid.

\section{A. Grid Connected Mode}

This will be the usual mode, in which the main objective of the intelligent connection agent is to track the grid voltage. In this mode, all the loads in the microgrid are being fed by the main grid or other power sources linked to the microgrid. Under these operating conditions, the VSI will take care of loading the dc-bus in order to keep the optimal state of charge.

In grid connected mode, the control loop drive the inverter as a voltage follower and feeds, or draws, the necessary active and reactive power to collaborate in maintaining the voltage and frequency values within limits set in the grid codes. An example of this application are the so-called grid-supporting inverters, which are designed to flexibly support the voltage and/or frequency of either a stand-alone or a interconnected grid, as shown in [6].

\section{B. Islanding Mode}

When a grid fault occurs, and it is detected, the VSI changes to work in island mode and the switch $S_{i}$, in Fig. 1, is opened. The microgrid VSI comes to act as a grid-forming converter and the energy is provided from an external dc source, which could be either a battery or another dc storage element. In this mode, the microgrid could be composed by other sources, for example, a current source inverter supplied

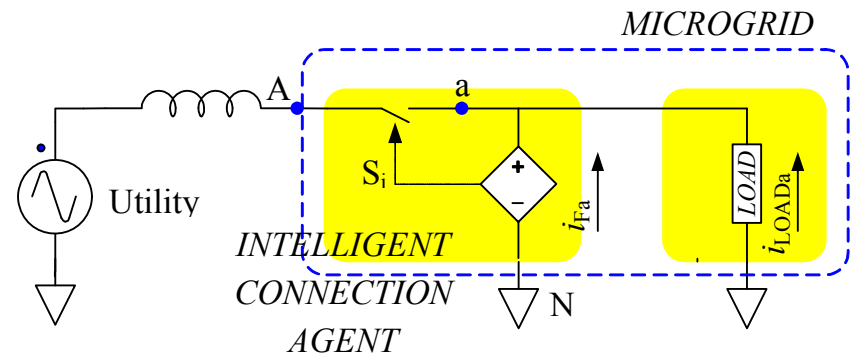

Fig. 1. Equivalent per phase network representation of the microgrid analised governed with the microgrid management agent.
PV panels. In this case, the VSI supplies or stores the necessary amount of energy to maintain the microgrid energy balance and keep frequency and voltage within the tolerance limits.

In the island mode, the control of the VSI is designed to act as a grid-forming element, with the objective of setting the grid voltage amplitude, frequency and phase. In the disconnection process the voltage amplitude and frequency will be the designed nominal values but the phase will be same that the system has got in the previous step in the switch turn-off. The main difference in the control scheme between the island and grid connected modes lies on the fact that in the island mode there is not any ac voltage available for starting the VSI voltage reference. Thus, the VSI himself generates the ac voltage of the microgrid. This voltage is sensed and supplied to the VSI controller as the main feedback signal. In the voltage control scheme presented in this paper, the microgrid sensed voltage is compared with the nominal voltage set point when the grid fault is detected and the island operation mode is triggered.

\section{Transient Operating Mode}

Under deficient grid voltage conditions, the main switch $\left(S_{i}\right)$ is turned off and disconnects the microgrid from the utility. This transient should be as fast as possible in order to ensure that the load on the microgrid do not experiment any significant transient while the power flow is maintained.

In a similar way to the connection of the islanding mode, another transient state arouses when the microgrid should reconnect to the network when operating as an island. Before reclosing the main switch $S_{i}$, the ICA should synchronize the phase-angle and the magnitude of the microgrid voltage with the voltage of the utility network. Once the microgrid is linked to the main network, the main switch is turn-on and VSI changes its operation mode to the grid-connected mode.

\section{CONTROL OF THE INTELLIGENT CONTROL AGENT}

In this section the control of the ICA will be explained when it is acting as either a voltage follower or a voltage former. In the first case, the main objective of the controller is to track the voltage of the mains, while in the second case voltage-forming is intended.

\section{A. Phase-Locked Loop (PLL)}

One of the most important aspects to be solved before connecting any generation system to the utility is to perform an accurate synchronization with the network voltage. In this work, a synchronization system based on a phase locked loop (PLL) has been implemented. The objective of this PLL is to obtain the phase angle $(\theta)$ of the three-phase ac grid voltage vector, which is expressed on a synchronous $d q$ reference frame.

The accurate estimation of this phase-angle allows both transforming the feedback variables into a synchronous reference frame, very suitable for control purposes of ac systems, and calculating and controlling the active/reactive power flow. 


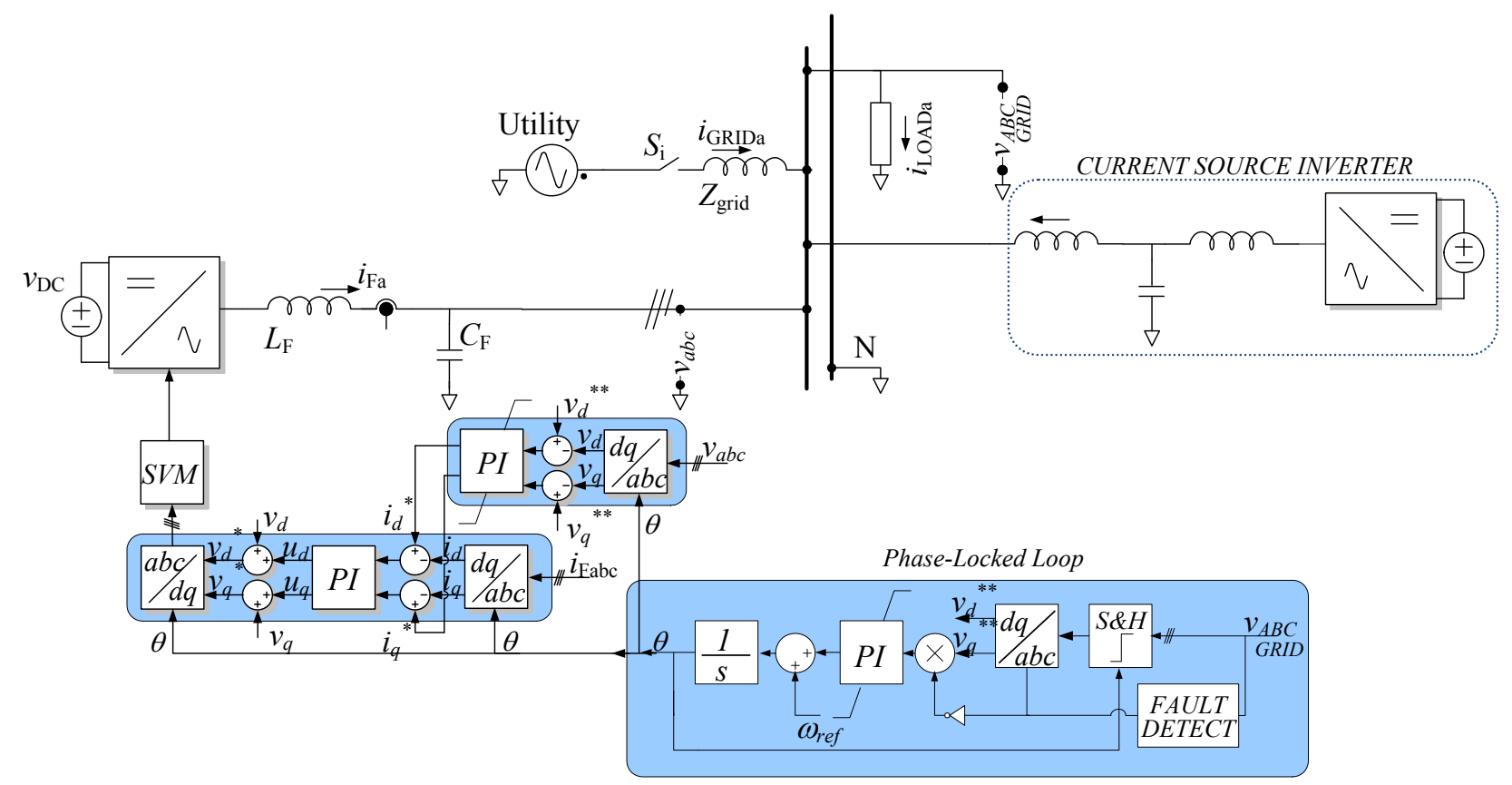

Fig. 2. Overall control blocks implementation of the proposed control diagram with the complete system. This scheme is implemented and simulated through co-simulation with MATLAB and PSIM.

The PLL used in this paper has been extracted from [7], [8], and a modified version it is shown in Fig.2. As it can be deduced from this figure, the measured three-phase grid voltage is transformed, using the Clark transformation, to the $\alpha \beta$ reference frame; then Park transformation is applied. This last transformation translates the $\alpha \beta$ ac variables to a synchronous $d q$ reference frame. This last step permits using conventional dc integrators to detect the phase-angle of the input voltage vector. As shown in Fig. 2, the quadrature component resulting from this synchronous transformation $\left(v_{q}\right)$ is conducted to zero by using a PI controller, thus obtaining the grid voltage positive sequence synchronized with the grid voltage. The output of this PI controller, added to a feedforward term representing the rated value of the grid frequency, is the rotating frequency of the synchronous reference frame. This frequency is integrated resulting in the phase-angle of the synchronous reference frame, $\theta$. When the PLL is perfectly synchronized, $v_{q}$ is equal to zero, which means that the phase-angle of the synchronous reference frame matches the phase-angle of the input voltage vector.

The dynamic of the PI controller determines the performance of the PLL. Therefore, the bandwidth of the system is a tradeoff between the PI filtering performance and the time response. So, it is important remember that this parameters of the loop, $k_{P}$ and $k_{I}$ constants of the PI controller has an influence both on the detection quality of the $\theta$ phaseangle and on the PLL dynamics. In this paper, slow PLL dynamics has been chose to attenuate the effect of the voltage harmonics on the grid synchronization process. However, if the synchronization algorithm would be used to detect grid faults, the system dynamics had to be increased

\section{B. Current and Voltage Control Loops}

The current and voltage control diagram of the ICA is shown in Fig.2. As it can be deduced from the figure, the voltage and the current control loops have been implemented using PI controllers working on the $d-q$ synchronous reference frame. An inner current control loop is formed around the filter inductor, and it is designed to have a very fast response. Moreover, an outer voltage control loop is in charge of tracking the grid voltage. The dynamics of this outer voltage loop is slower than the one for the current loop. This grid-follower control works as an observer of the main grid voltage and it is also responsible of maintaining the voltage reference when the ac bus of the microgrid is

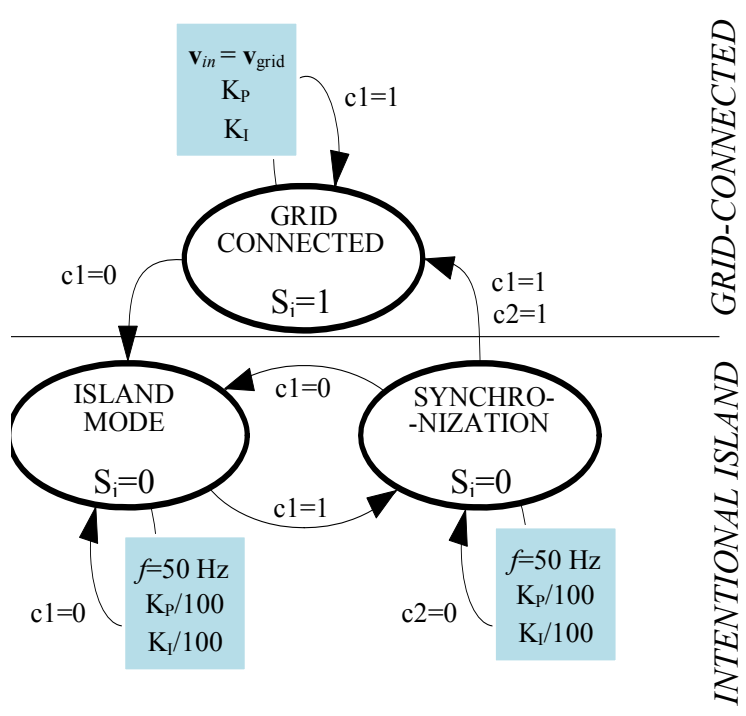

Fig. 3. The upper diagram shows the algorithm process in the different operating cases are defined in function of environment condition. 
disconnected from the utility, [9].

\section{Island Mode and Resynchronization Mode}

There are a lot of references in which some anti-island techniques are proposed. In these references active and passive schemes are shown. In our case, a passive technique through the measurements of the grid module and grid frequency is used. When an out of bounds situations is done in the voltage values or in frequency measurement is done, instantaneously the main switch is opened and the control is switched to voltage mode. The Intentional Island situation is forced and the VSI injects the required power to maintain the instantaneous voltage to a given reference with a specified voltage and frequency. The VSI is designed basically to give the required energy to maintain the voltage waveform during the transient between both modes, and when connections or disconnections transients are done in Island mode. The first condition, $\mathrm{C} 1$, to pass to Island mode is to get into the minimum and maximum allowed values, defined in the standards.

When the microgrid works in island mode, the PLL will be disabled. In this mode, the voltage and frequency will be defined as a design factor. When the main grid voltage was restored the PLL initialize the resynchronization process. The PLL voltage input will be enable again. So, under any phase difference between both phase angles, a quadrature measurement in the PLL voltage error will appear. The PI controller, inside the PLL, slowly moves the microgrid phase angle reference until eliminate any quadrature component in $\mathrm{d}-\mathrm{q}$ voltages. In this case, the microgrid still works in island.

A fast PLL performance is necessary in grid-connection mode, but in island the opposite effect is required. A slow synchronization is essential, with the objective to minimize the frequency perturbation with a slow phase modification. This change in the dynamics of the PLL is done through the modification of the inner $k_{P}$ and $k_{I}$ constants of the PI controller. In Island mode this values are divided per one hundred. Loads need to have a constant frequency, plus or minus a tolerance band. When the error in the quadrature voltage is conducted to zero, the synchronization with the main grid is done. The second condition, C2, needs a near zero values of the quadrature signal to be true. When both conditions, $\mathrm{C} 2$ and $\mathrm{C} 1$, are true then the ICA could be closed again. It is important mention that in resynchronization process only an unique PLL structure is required, moved by the grid voltage. This case algorithm is explained in Fig.3.

\section{Simulation Results}

The performance of the whole system with the proposed control has been simulated, shown in Fig. 2. The simulated system is composed by the utility grid, with the line impedance and an active switch governed by the supervisor control. This switch is used to connect, or disconnect, the microgrid to the main grid. This ac bus is also responsible of feeding the loads. In this case the load is purely resistive.
Also a CSI is added simulating any power source feeding the grid.

The model has been implemented in co-simulation with Simulink/MATLAB and PSIM. The environment and the results are shown in the figures below, from Fig. 4 to Fig. 7. The simulation parameters used are given in Table I.

TABLE I

SIMULATION PARAMETERS

\begin{tabular}{lcc}
\hline \hline \multicolumn{1}{c}{ Parameter } & Symbol & Value and Units \\
\hline Filter Inductance & $L_{F}$ & $2.47 \mathrm{mH}$ \\
Filter Capacitance & $C_{F}$ & $20 \mu \mathrm{F}$ \\
dc voltage source & $V_{D C}$ & $700 \mathrm{~V}$ \\
Grid Inductance & $L_{G R I D}$ & $1 \mathrm{mH}$ \\
Grid Resistance & $R_{G R I D}$ & $0.5 \Omega$ \\
Resistive Load & $R_{L O A D}$ & $20 \Omega$ \\
Voltage Loop PI constant values & $k_{P}, k_{I}$ & $0.02,10$ \\
Current Loop PI constant values & $k_{P,}, k_{I}$ & 20,100 \\
\hline \hline
\end{tabular}

In this simulation the system starts to operate in a gridconnected mode and the load is fully fed by the grid, while the VSI only tracks the grid voltage. Then, at $\mathrm{t}=0.1 \mathrm{~s}$, an ideal and symmetrical voltage dip of $50 \%$ is applied at the point of common coupling (PCC), simulating a fault in the utility distribution grid. At $\mathrm{t}=0.15 \mathrm{~s}$ the voltage utility grid is restored again, but with a significant change with the phase angle, so this reconnection is not done yet. First a resynchronization phase in which the microgrid ac bus slowly change to utility grid phase, according to designed $\mathrm{Hz} / \mathrm{s}$ ratios. At $\mathrm{t}=0.499 \mathrm{~s}$ when both phase angle are nearly equal and the physical reconnection can be done. These results are shown in Fig. 4, and zoom it in Fig. 5.

When the system is operating in island mode and the main network is considered recovered, the synchronization process starts. For microgrid voltage resynchronization the utility voltage quadrature component is input in the PI of the PLL. This option would ensure that the microgrid voltage phase to be synchronized with utility voltage phase. This quadrature evolution is represented in Fig. 6.

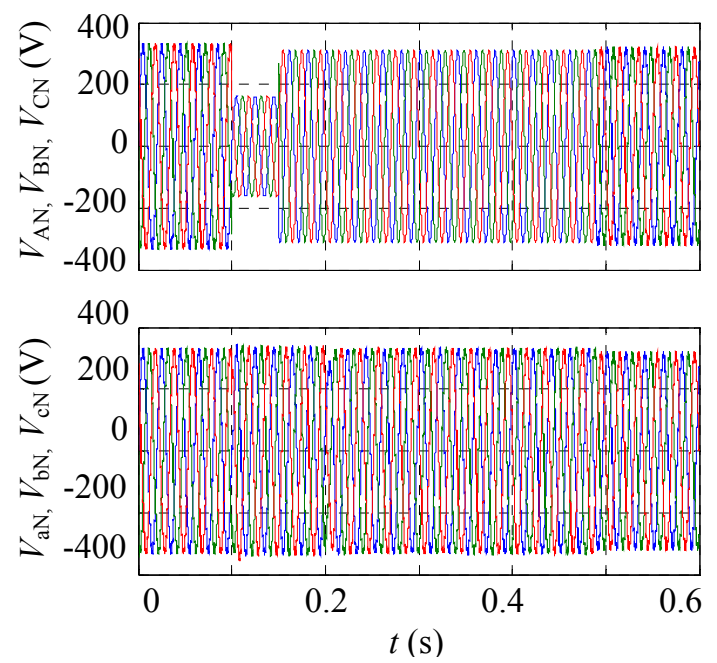

Fig. 4. The upper diagram shows the grid voltage waveform during a symmetrical grid fault applied on three voltage grid phase and its restoration after some cycles. 
The CSI follows a square wave power profile reference between 0 and $2 \mathrm{~kW}$, as shown in the middle graph of Fig. 7. Also the microgrid includes a resistive load which consumes $2 \mathrm{~kW}$ nominal power. The network will deliver the power difference between CSI and the load, when it is available. Otherwise, when microgrid is disconnected from the main network, will be the VSI who delivers the energy needed to feed the load.

Fig. 7 shows the evolution of instant active power delivered by each element during the simulation. In the above graph is checked as the load does not perceive a significant variation of the power absorbed by this under the different transients. The middle graph shows the power delivered by the CSI, this follows a square power pattern generated by an external reference. The chart below shows the power injected by the VSI and the input power at the PCC, the grid power.

\section{CONCLUSION}

This paper proposes a control strategy of a voltage source inverter to operate as a microgrid Intelligent Connection Agent to form a secure ac bus. This proposed agent has been programmed to control the disconnection or reconnection to the main electrical grid, giving rise to excellent results.

The simulations results had shown the good performance and a fast dynamics of the proposed system so in disconnection transient as in resynchronization and reconnection. Also, in grid-connected mode the proposed controller is capable to follow the voltage grid, in amplitude, frequency and phase. In islanding mode, the ICA presents a good regulation feeding the loads with a specific voltage, frequency and phase.

It is important mention that, in resynchronization process only one PLL structure is required, improving the performance and simplifying the computational cost.
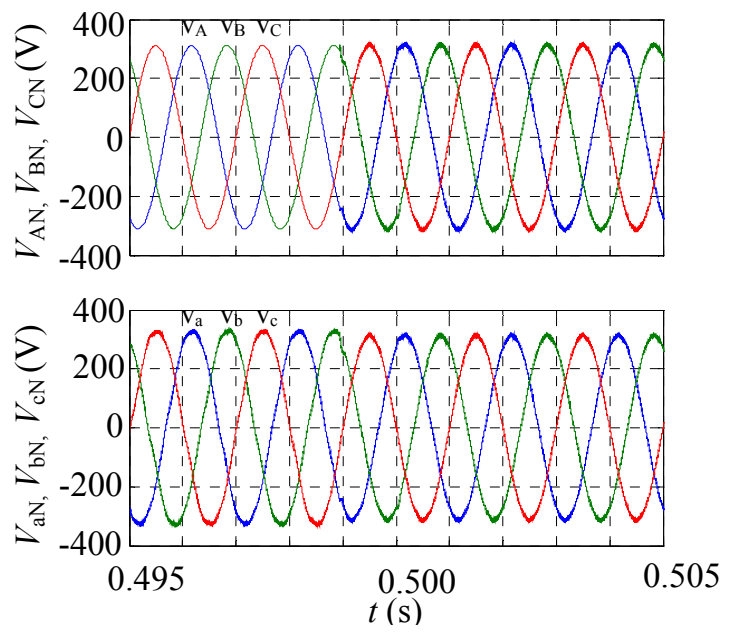

Fig. 5. Detailed view of the full simulation in Fig.3, in the upper diagrams the utility grid voltage is represented while the lower ones show the microgrid voltage. In the first column at $\mathrm{t}=0.1 \mathrm{~s}$ disconnection transient is done due a voltage sag in the main voltage grid. At $\mathrm{t}=0.15$ the main grid voltage is restored but phase difference does not allow the reconnection, yet. In the second column the final part of the resynchronization and the reconnection transient is presented when the three phases are synchronized. The connection is done in $\mathrm{t}=0.499$.

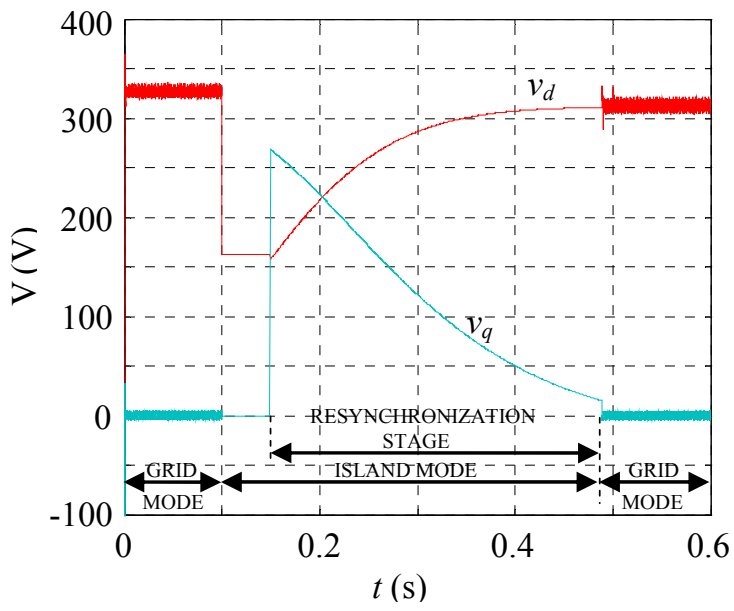

Fig. 6. Temporal direct and quadrature voltage evolution in different microgrid modes.
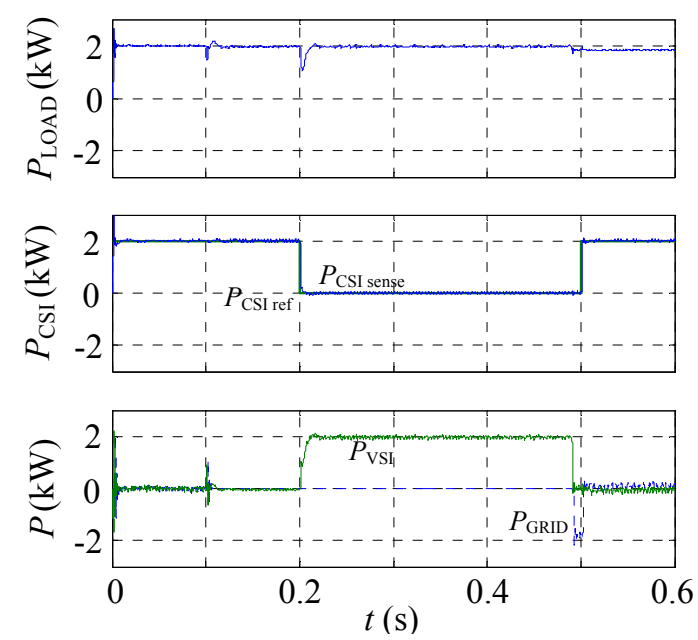

Fig. 7. Temporal power evolution delivered by each microgrid element. In the top chart shows the power absorbed by the load; in the middle graph shows the power delivered by CSI, whereas in the chart below shows the power delivered by the VSI and the grid. 


\section{ACKNOWLEDGMENT}

This work was supported by the Ministerio de Ciencia y Tecnología, Madrid, Spain, under Grant ENE2008-06841C02-01/ALT.

\section{REFERENCES}

[1] Z. Ye, A. Kolwalkar, Y. Zhang, P. Du, and R. Walling, "Evaluation of anti-islanding schemes based on nondetection zone concept," Power Electronics, IEEE Transactions on, vol. 19, pp. 1171-1176, 2004.

[2] M. Liserre, A. Pigazo, A. Dell'Aquila, and V. M. Moreno, "An AntiIslanding Method for Single-Phase Inverters Based on a Grid Voltage Sensorless Control," Industrial Electronics, IEEE Transactions on, vol. 53, pp. 1418-1426, 2006.

[3] H. Karimi, A. Yazdani, and R. Iravani, "Negative-Sequence Current Injection for Fast Islanding Detection of a Distributed Resource Unit," Power Electronics, IEEE Transactions on, vol. 23, pp. 298-307, 2008.

[4] J. M. Guerrero, N. Berbel, J. Matas, J. L. Sosa, and L. G. de Vicuna, "Control of Line-Interactive UPS Connected in Parallel Forming a Microgrid," presented at Industrial Electronics, 2007. ISIE 2007. IEEE International Symposium on, 2007.

[5] T. Thacker, F. Wang, and D. Boroyevich, "Islanding Control of a Distributed Generation Unit's Power Conversion System to the Electric Utility Grid," in Proc. IEEE 36th Power Electronics Specialists Conference, 2005. PESC '05, 2005.

[6] S. J. Chiang, C. Y. Yen, and K. T. Chang, "A multimodule parallelable series-connected PWM voltage regulator," Industrial Electronics, IEEE Transactions on, vol. 48, pp. 506-516, 2001.

[7] A. Timbus, R. Teodorescu, F. Blaabjerg, and M. Liserre, "Synchronization Methods for Three Phase Distributed Power Generation Systems. An Overview and Evaluation," presented at Power Electronics Specialists Conference, 2005. PESC '05. IEEE 36th, 2005.

[8] V. Kaura and V. Blasko, "Operation of a phase locked loop system under distorted utility conditions," Industry Applications, IEEE Transactions on, vol. 33, pp. 58-63, 1997.

[9] J. M. Guerrero, L. Hang, and J. Uceda, "Control of Distributed Uninterruptible Power Supply Systems," Industrial Electronics, IEEE Transactions on, vol. 55, pp. 2845-2859, 2008. 